\section{Welche Faktoren beeinflussen Depression und Angst bei Angehörigen unheilbar Tumorkranker?}

\begin{abstract}
Angehörige oder Freunde, die sich um einen unheilbar Krebskranken kümmern, sind wichtig für sein Wohlergehen. Die hohen Anforderungen können bei Bezugspersonen zu deutlicher psychischer Belastung führen.
\end{abstract}

nsgesamt 275 Patienten, bei denen maximal 8 Wochen zuvor die Primärdiagnose eines unheilbaren Lungen- oder gastrointestinalen Tumors gestellt worden war und die noch keine Tumortherapie erhalten hatten, wurden in eine Studie

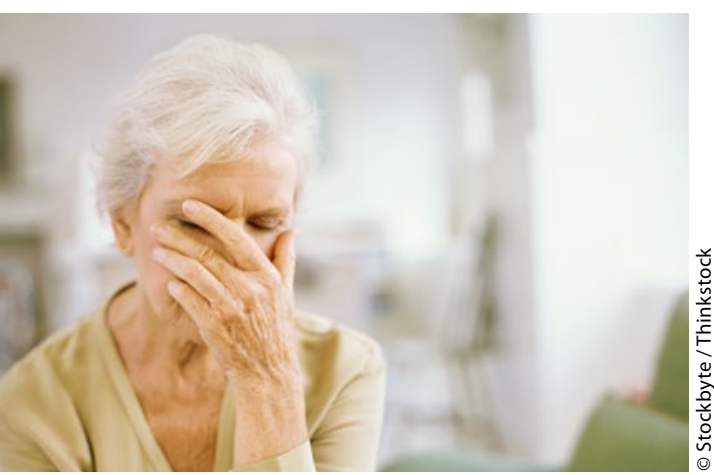

Manche Copingstrategien von Tumorpatienten belasten die Angehörigen. aufgenommen. Voraussetzung war, dass sie einen Angehörigen oder Freund nennen konnten, der zur Mitarbeit bereit war und sie zur Sprechstunde begleitete. Geprüft wurden Depression und Angst beim Patienten selbst und bei den Angehörigen/Freunden, außerdem Lebensqualität und Copingstrategien des Patienten. Zudem wurden die Patienten nach dem primären Ziel der Therapie befragt.

Depressionen waren bei Bezugspersonen und Patienten gleich häufig (16,4 vs. $21,5 \% ; p=0,13)$, Bezugspersonen berichteten aber signifikant häufiger über Angst $(42,2$ vs. $28,4 \%$; $p<0,001)$. Das könnte z.B. damit zusammenhängen, dass die Betreuenden Sorge haben, sie könnten den auf sie zukommenden Anforderungen nicht gewachsen sein. Die Copingstrategien der Patienten wirkten sich deutlich auf das Befinden der Angehörigen aus. Setzten die Patienten bevorzugt die Copingstrategie „Akzeptanz“ ein, war die Neigung der Betreuungspersonen zu Depression geringer $(\mathrm{p}<0,001)$. Dagegen ging die an sich wünschenswerte Strategie „emotionale Unterstützung“ bei den Angehörigen verstärkt mit Depression einher ( $\mathrm{p}=0,001)$, während sie gleichzeitig die Neigung zu Angst verringerte $(\mathrm{p}<0,001)$. Das bedeutet: Geben Angehörige und Freunde emotionale Unterstützung, kann das zwar die Angst mindern, hinterlässt aber bei den Angehörigen selbst ein Gefühl von Traurigkeit. Die Einschätzung der Prognose durch den Patienten selbst spielt auch eine wichtige Rolle. Ein Drittel gab als primäres Behandlungsziel „Heilung“ an. Dies war bei den Bezugspersonen häufiger mit Depression assoziiert $(\mathrm{p}=0,03)$.

Fazit: Patienten mit unheilbarer Tumorerkrankung und ihre Betreuungspersonen leiden häufig an Depression und Angst. Die Copingstrategien der Patienten und ihre Einschätzung der Prognose haben Einfluss auf Angst und Depression bei den Bezugspersonen. Brigitte Schalhorn

Nipp RD et al. Factors associated with depression and anxiety symptoms in family caregivers of patients with incurable cancer. Ann Oncol. 2016;27(8):1607-12.

\section{Toxizität der Chemotherapie bei Älteren mithilfe eines praxisgerechten Scores abschätzen}

\author{
Bekannte Instrumente wie Karnofsky-Performancestatus (PS) oder ECOG-PS \\ sind nicht speziell für ältere Menschen validiert. Daher wurde bei 500 älteren \\ Patienten mit soliden Tumoren ein Vorhersagemodell entwickelt und nun \\ extern validiert, um die Toxizität einer Chemotherapie vorauszusagen.
}

$\mathrm{D}$ as prädiktive Vorhersagemodell zur chemotherapiebedingten Toxizität bei älteren Krebspatienten wurde in einer externen Kohorte mit 250 älteren $(\geq 65$ Jahre) Patienten mit soliden Tumoren validiert. Das Modell beruhte auf 11 Parametern, sowohl aus der täglichen onkologischen Routine (z. B. Alter, Tumortyp, geplante Dosierung, Kreatinin-Clearance) als auch aus dem geriatrischen Assessment (z.B. Hörvermögen, Anzahl von Stürzen in den letzten 6 Monaten, selbstständige Tabletteneinnahme) [Hur- ria A et al. J Clin Oncol. 2011;29(25):345765]. Das Risiko chemotherapiebedingter Toxizitäten wurde vor Behandlungsbeginn auf Basis des Modells kalkuliert (niedrig, mittel oder hoch). Anschließend wurde der Schweregrad der aufgetretenen Nebenwirkungen protokolliert (Grad 3-5). Die Validierung des Vorhersagemodells erfolgte mit der C-Statistik (ROCKurve). Am häufigsten litten die Patienten an Brustkrebs, an Tumoren der Lunge oder des Gastrointestinaltraktes. 52\% hatten eine Erkrankung im Stadium IV,
$70 \%$ erhielten eine Polychemotherapie, meist in der Firstline. $58 \%$ erfuhren eine Toxizität vom Grad $\geq 3$. Mit steigendem Risikoscore nahmen unerwünschte Wirkungen signifikant zu (niedrig: $36,7 \%$, mittel: $62,4 \%$, hoch: $70,2 \% ; p<0,001)$. In der ROC-Statistik unterschieden sich die Werte der externen Validierungskohorte nicht von denen der Entwicklungskohorte. Es ergab sich keine signifikante Assoziation zwischen Karnofsky-PS und chemotherapiebedingter Toxizität $(\mathrm{p}=0,25)$.

Fazit: Das von Arti Hurria und Kollegen entwickelte Vorhersagemodell für die Toxizität einer Chemotherapie bei älteren Menschen in der täglichen Praxis erwies sich auch in der Validierung als praktikabel. Brigitte Schalhorn

Hurria A et al. Validation of a prediction tool for chemotherapy toxicity in older adults with cancer. J Clin Oncol. 2016;34(20):2366-71. 